\title{
CLIV. THE ANTIHAEMORRHAGIC VITAMIN OF THE CHICK.
}

\author{
BY HENRIK DAM. \\ From the Biochemical Institute, University of Copenhagen.
}

(Received April 8th, 1935.)

Previous papers [Dam, 1929; 1930; Dam and Schönheyder, 1934] deal with a deficiency disease resembling scurvy in chicks which cannot be prevented by ascorbic acid and the cause of which is ascribed to the lack of a particular antihaemorrhagic factor (or factors) in the diet. Schönheyder [1935] has shown that there is an enormous retardation of the clotting of the blood of chicks suffering from this haemorrhagic diathesis.

The nature and distribution of the antihaemorrhagic factor have now been investigated. The investigation has led to the discovery of the fact that the factor is a fat-soluble vitamin occurring in hog-liver, hemp seed, certain cereals and vegetables, and must be different from vitamins $\mathrm{A}, \mathrm{D}$ and $\mathrm{E}$. It is proposed to term this factor vitamin $K$ (Koagulations-Vitamin in German and the Scandinavian languages).

The following groups of foods have been tested: (1) cereals and seeds, (2) vegetables, (3) animal organs, (4) different fats and oils, (5) hen's egg.

Two of the most active substances, hog-liver and hemp seed, were divided into ether-soluble and ether-insoluble fractions, and, since the active principle was found to be fat-soluble, an elaborate fractionation of hog-liver fat was carried out. The question of the identity of the antihaemorrhagic factor with already known fat-soluble vitamins has been attacked by adding large amounts of vitamins $\mathrm{A}, \mathrm{D}$ and $\mathrm{E}$ to the basal diet.

\section{EXPERIMENTAL.}

The animals (White Leghorns) were fed the experimental diets from the day after hatching. They were, as a rule, killed at the age of about 1 month. Housing etc. as described by Dam and Schönheyder [1934].

The examination of the animals includes, in addition to an inspection of the gizzard and other organs for haemorrhages, a determination of the time of clotting of the blood. The haemoglobin content of the blood has also been determined because it may give some information about the loss of blood through the haemorrhages.

The haemoglobin values were determined by the Sahli haemoglobinometer; the figures indicate the haemoglobin content in percentage of that in normal human blood.

The clotting time was determined by opening the brachial vein by a slight cut and allowing 2-3 ml. blood to drop slowly, during $\frac{1}{2}-\frac{3}{4} \mathrm{~min}$., into a small porcelain bowl. The clotting time is reckoned from the venepuncture until complete clotting ${ }^{1}$.

1 Autopsy and clotting time determinations were kindly made by Dr F. Schönheyder to whom the author is indebted. Dr Schönheyder has in the meantime developed a new technique which enables the quantitative determination of the antihaemorrhagic vitamin to be made by a curative method based on a particularly exact measurement of the clotting time [1935]. The results presented in this paper were obtained by means of the former approximate method but give fairly accurate information about the degree of sickness of the animals. 
Basal diets.

\begin{tabular}{|c|c|c|c|c|c|}
\hline \multicolumn{2}{|l|}{60} & \multicolumn{2}{|l|}{111} & \multicolumn{2}{|l|}{124} \\
\hline $\begin{array}{l}\text { Caseinogen } \\
\text { vitamin A-free }\end{array}$ & 20 & $\begin{array}{l}\text { Caseinogen } \\
\text { vitamin A-free }\end{array}$ & 20 & $\begin{array}{r}\text { Ether-extracted } \\
\text { dried hog-liver }\end{array}$ & 20 \\
\hline Dried yeast & 15 & Dried yeast & 40 & Dried yeast & 15 \\
\hline Salts No. $2^{*}$ & $2 \cdot 7$ & Salts Ňo. 2 & $2 \cdot 7$ & Salts Ňo. 2 & $2 \cdot 7$ \\
\hline Sucrose & $62 \cdot 3$ & $\begin{array}{l}\mathrm{CaCO}_{3} \\
\text { Sucrose }\end{array}$ & $\begin{array}{r}0.85 \\
36 \cdot 45 \\
\end{array}$ & Sucrose & $62 \cdot 3$ \\
\hline & 100 & & 100 & & 100 \\
\hline Cod-liver oil & 4 & Cod-liver oil & 4 & Cod-liver oil & 4 \\
\hline
\end{tabular}

Cereals and seeds were crushed and given instead of sucrose in diet 60 (with a small amount of $\mathrm{CaCO}_{3}$ added roughly to balance the excess $\mathrm{P}_{2} \mathrm{O}_{5}$ in the cereals). Organs were dried in a current of air at a temperature not exceeding $45^{\circ}$ and given as a powder instead of caseinogen in diet 60 . Vegetables were minced and mixed into the diet or given in separate food containers. Vitamin concentrates: "vogan" (a vitamin A preparation from Merck, Darmstadt with 120,000 International Units per ml.), "vigantol" (pure vitamin D in sesame oil, from Merck, with 15,000 I.U. per ml.) and carotene (Hoffmann La Roche, Basel) dissolved in olive oil, were given per os by means of a tuberculin syringe, the doses being gradually increased from week to week. Vigantol was diluted with olive oil because the day doses otherwise would have been too small for accurate measurement with the syringe.

Hog-liver fat was put at the author's disposal by the firm of Medicinalco, Copenhagen. It was, according to the statement of the manufacturer, prepared by percolation of the desiccated liver with light petroleum several times, and thereafter with ether. The solvents were removed in vacuo. The percolated liver was used in diet 124 after having been further extracted with ether in a Soxhlet apparatus for 3 days. Hemp seed oil was prepared by percolating the crushed seeds with ether, and the seeds were further extracted with ether for several days before they were used in the diet as "ether-extracted hemp seed". Wheat germ oil was prepared from the fresh germ which had just been dried in air at $35^{\circ}$. The extraction was carried out in a large Soxhlet apparatus within 8 hours, the bulk of the ether was distilled off in $\mathrm{CO}_{2}$ and the rest removed in vacuo. All oils were stored in the ice-box in completely filled containers and the liver fat in vacuo. The oils were used within 2 months of the day of preparation. The ether was in all cases freshly distilled and tested with mercury before use. Non-saponifiable matter of liver fat was prepared by cold saponification of $100 \mathrm{~g}$. of the fat in $250 \mathrm{ml}$. ether with $40 \mathrm{~g}$. KOH in $250 \mathrm{ml}$. methyl alcohol for 16-24 hours, addition of water and shaking 3-4 times with more ether; the ether extracts were shaken 3 times with aqueous $\mathrm{KOH}$ and then with water to neutral reaction. The extraction and purification of the ether extract were carried out in one day (in the dark room). After standing overnight in the ice-box with $\mathrm{Na}_{2} \mathrm{SO}_{4}$ and filtering, the solution was concentrated (in $\mathrm{CO}_{2}$ and then in vacuo) to $100 \mathrm{ml}$. On standing overnight in the ice-box a precipitate of an amorphous substance, which is soluble in ether, could be filtered off. In the experiments in which the sterol and non-sterol fractions were tested, the bulk of the sterols was crystallised from light petroleum (after evaporation of the filtrate from the readily soluble substance). The rest of the sterols were precipitated by digitonin in small excess. The digitonin precipitate was decomposed by the pyridine-ether method of Schönheimer and Dam [1933] in the cold, and the sterol prepared in this way was added to the crystallised main portion. The 
filtrate from the digitonide was brought to dryness in vacuo and extracted with ether which takes up the "easily soluble non-sterol fraction".

The fatty acids were precipitated from the extracted soaps by sulphuric acid after evaporation of the alcohol in vacuo; the acids were washed with water by decantation.

In one experiment the non-saponifiable matter was divided into a "carotene" fraction and a "xanthophyll" fraction by partitioning between light petroleum and $90 \%$ (by volume) methyl alcohol, after removing the sterols as far as possible by crystallisation.

Table I.

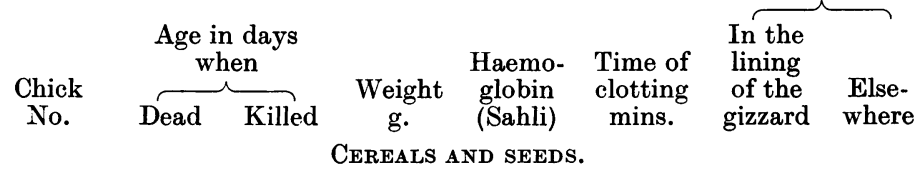

Group 75. $62 \%$ whole wheat:

$\begin{array}{llllllll}564 & - & 31 & 127 & 45 & - & + & 0 \\ 565 & - & 31 & 318 & 65 & - & 0 & 0 \\ 566 & - & 31 & 240 & 51 & - & + & 0 \\ 567 & - & 16 & 87 & 59 & - & ? & 0 \\ 568 & - & 31 & 217 & 59 & - & + & 0\end{array}$

Group 77. $62 \%$ wheat meal:

$\begin{array}{llllllll}574 & - & 16 & 73 & 48 & - & + & 0 \\ 575 & - & 31 & 212 & 45 & - & + & 0 \\ 576 & - & 31 & 224 & 39 & - & + & 0 \\ 577 & - & 29 & 175 & 37 & - & + & ++ \\ 578 & - & 11 & 83 & 48 & - & + & +\end{array}$

Group 79. $62 \%$ wheat bran:

$\begin{array}{llllllll}\mathbf{5 8 4} & - & 31 & 107 & 49 & - & 0 & 0 \\ \mathbf{5 8 5} & - & 31 & 139 & 54 & - & 0 & 0 \\ \mathbf{5 8 6} & - & 16 & 55 & \mathbf{7 0} & - & 0 & 0 \\ \mathbf{5 8 7} & - & 31 & 151 & \mathbf{5 2} & - & 0 & 0 \\ \mathbf{5 8 8} & - & 31 & 133 & \mathbf{5 7} & - & 0 & 0\end{array}$

Group 86. $62 \%$ wheat germ :

$\begin{array}{llrrllll}619 & 7 & - & 30 & - & - & 0 & 0 \\ 620 & - & 28 & 174 & 66 & - & 0 & + \\ 621 & - & 7 & 21 & - & - & ? & 0 \\ 622 & - & 33 & 154 & 55 & - & 0 & + \\ 623 & - & 38 & 183 & 55 & 19 & + & 0 \\ 699 & - & 16 & 54 & - & - & + & 0 \\ 700 & - & 30 & 218 & \tilde{59} & 2 & ? & 0\end{array}$

Group 93. $62 \%$ oats:

$\begin{array}{llllllll}654 & - & 33 & 412 & 51 & 2 & 0 & 0 \\ 655 & - & 33 & 442 & 62 & 1 & ? & ? \\ 656 & - & 33 & 315 & 62 & 1 & 0 & 0 \\ 657 & 5 & - & 29 & - & - & 0 & 0 \\ 658 & - & 28 & 234 & 50 & 3 \frac{1}{2} & 0 & +\end{array}$

Group 94. $62 \%$ yellow corn:

$\begin{array}{lllllrrr}659 & - & 30 & 135 & 42 & 20 & + & + \\ 660 & - & 33 & 232 & 46 & - & + & + \\ 661 & - & 33 & 179 & 65 & 3 & + & 0 \\ 662 & - & 33 & 169 & 35 & >12 & + & + \\ 663 & - & 33 & 195 & 55 & 2 & + & 0\end{array}$

Group 95. $62 \%$ barley :

\begin{tabular}{|c|c|c|c|c|c|c|}
\hline 664 & - & 33 & 285 & $\tilde{55}$ & $\frac{1}{2}$ & 0 \\
\hline 665 & - & 33 & 274 & 58 & $1^{2}$ & 0 \\
\hline 666 & - & 33 & 284 & 60 & $\frac{1}{2}$ & 0 \\
\hline 667 & - & 33 & 225 & 50 & 1 & $0 ?$ \\
\hline 668 & - & 33 & 218 & 58 & $\frac{1}{2}$ & 0 \\
\hline
\end{tabular}


Table I (cont.).

Haemorrhages

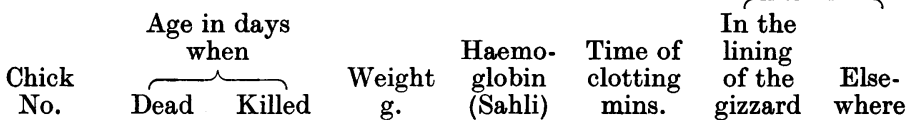

Group $96.62 \%$ millet:

$\begin{array}{llllllll}669 & - & 33 & 312 & 60 & 2 \frac{1}{2} & ? & 0 \\ 670 & - & 33 & 290 & 61 & 1 & 0 & + \\ 671 & - & 33 & 248 & 61 & \frac{1}{2} & 0 & 0 \\ 672 & - & 33 & 247 & 60 & 1^{\frac{1}{2}} & 0 & 0 \\ 673 & - & 33 & 304 & 65 & 1 & + & 0\end{array}$

Group 97. $62 \%$ hemp seed:

\begin{tabular}{|c|c|c|c|c|c|c|c|}
\hline 674 & - & 33 & 258 & 50 & $\frac{1}{2}$ & 0 & 0 \\
\hline 675 & - & 33 & 263 & 60 & 1 & 0 & 0 \\
\hline 676 & - & 33 & 313 & 61 & $\frac{1}{2}$ & 0 & 0 \\
\hline 677 & - & 33 & 310 & 60 & $\frac{1}{2}$ & 0 & 0 \\
\hline 678 & & 33 & 310 & 50 & 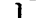 & 0 & 0 \\
\hline
\end{tabular}

Group 98. $62 \%$ unpolished rice:

$\begin{array}{lrrrrrrr}679 & 7 & - & - & - & - & - & - \\ 680 & - & 20 & 154 & 32 & >8 & + & ++ \\ 681 & - & 30 & 145 & 45 & >12 & + & + \\ 682 & - & 15 & 75 & 27 & >120 & + & + \\ 683 & - & 13 & 86 & 40 & >18 & + & +\end{array}$

Group 146. $62 \%$ white sunflower seed:

$\begin{array}{lllllcll}928 & - & 31 & 245 & 55 & >37 & + & 0 \\ 929 & - & 31 & 198 & 58 & 30 & + & 0 \\ 930 & - & 31 & 275 & 55 & 1 \frac{1}{2} & + & 0 \\ 931 & - & 31 & 300 & 55 & 100 & + & + \\ 932 & - & 31 & 260 & 58 & 5 & 0 & +\end{array}$

Group 147. $62 \%$ black sunflower seed:

$\begin{array}{lllrlrrr}933 & 13 & - & 68 & - & - & + & ++ \\ 934 & - & 23 & 139 & 55 & >130 & 0 & ++ \\ 935 & - & 16 & 77 & 10 & >60 & + & ++ \\ 936 & - & 23 & 146 & 46 & >120 & + & + \\ 937 & - & 23 & 169 & 58 & >195 & 0 & 0\end{array}$

Group 149. $62 \%$ soya bean:

$\begin{array}{llllllll}943 & - & 33 & 198 & 53 & \frac{1}{2} & ? & 0 \\ 944 & - & 33 & 147 & 55 & 2 \frac{1}{2} & 0 & 0 \\ 945 & - & 33 & 189 & 49 & 2 \frac{1}{2} & + & 0 \\ 946 & - & 33 & 228 & 50 & 1 & + & 0 \\ 947 & - & 33 & 151 & 48 & 2 \frac{1}{2} & + & ?\end{array}$

Group 173. $62 \%$ rye :

$\begin{array}{llllllll}1070 & - & 44 & 260 & 42 & 110 & + & + \\ 1071 & - & 32 & 220 & 55 & 9 & + & 0 \\ 1072 & 48 & - & 345 & - & - & + & 0 \\ 1073 & - & 56 & 467 & 54 & 52 & + & 0 \\ 1074 & - & 44 & 265 & - & - & + & 0\end{array}$

Fractions of hemp Seed.

Group 120. $62 \%$ ether-extracted hemp seed:

$\begin{array}{llrrrrrr}801 & - & 25 & 63 & 50 & 3 \frac{1}{2} & + & 0 \\ 802 & - & 24 & 52 & 25 & 4 & + & ? \\ 803 & 19 & - & 42 & - & - & + & 0 \\ 804 & - & 9 & 36 & 62 & 2 \frac{1}{2} & 0 & ? \\ 805 & - & 25 & 143 & - & - & + & 0\end{array}$

Group 132. Diet $111+25 \%$ hemp seed oil:

$\begin{array}{llllllll}862 & - & 42 & 298 & 50 & 2 & 0 & 0 \\ 863 & - & 42 & 265 & 60 & 2 & 0 & 0 \\ 864 & - & 43 & 310 & 55 & 1 & ? & 0 \\ 865 & - & 39 & 169 & 45 & 1 & ? & 0 \\ 866 & - & 33 & 150 & 60 & 1 & ? & 0\end{array}$


Table I (cont.).

\begin{tabular}{|c|c|c|c|c|c|c|c|}
\hline \multirow{3}{*}{$\begin{array}{l}\text { Chick } \\
\text { No. }\end{array}$} & \multirow{2}{*}{\multicolumn{2}{|c|}{$\begin{array}{l}\text { Age in days } \\
\text { when }\end{array}$}} & \multirow{3}{*}{$\begin{array}{l}\text { Weight } \\
\text { g. }\end{array}$} & \multirow{3}{*}{$\begin{array}{l}\text { Haemo- } \\
\text { globin } \\
\text { (Sahli) }\end{array}$} & \multirow{3}{*}{$\begin{array}{l}\text { Time of } \\
\text { clotting } \\
\text { mins. }\end{array}$} & \multicolumn{2}{|c|}{ Haemorrhages } \\
\hline & & & & & & $\begin{array}{l}\text { In the } \\
\text { lining }\end{array}$ & \\
\hline & Dead & Killed & & & & $\begin{array}{l}\text { of the } \\
\text { gizzard }\end{array}$ & $\begin{array}{l}\text { Else- } \\
\text { where }\end{array}$ \\
\hline
\end{tabular}

Group 111. Diet 111 without any addition:

$\begin{array}{llllllll}751 & - & 52 & 308 & 35 & 120 & + & + \\ 752 & - & 52 & 309 & 50 & 6 & + & + \\ 753 & 7 & - & - & - & - & 0 & 0 \\ 754 & - & 42 & 212 & 32 & >26 & + & + \\ 755 & - & 19 & 114 & 36 & >60 & 0 & + \\ 756 & - & 45 & 170 & 43 & >60 & + & + \\ 757 & - & 33 & 148 & 25 & >60 & + & +\end{array}$

Vegeta BLES.

Group 84. Diet $60+25 \%$ fresh kale:

$\begin{array}{lrrrrrrr}609 & - & 9 & 30 & 57 & - & 0 & 0 \\ 610 & - & 38 & 218 & 54 & - & 0 & 0 \\ 611 & - & 38 & 279 & 51 & - & 0 & 0 \\ 612 & - & 32 & 100 & 70 & - & 0 & 0 \\ 613 & - & 38 & 200 & 51 & - & 0 & 0\end{array}$

Group 118. Diet 60 in which $15 \%$ sugar is replaced by dried alfalfa:

\begin{tabular}{|c|c|c|c|c|c|c|}
\hline 788 & - & 35 & 249 & 52 & $5 \frac{1}{2}$ & 0 \\
\hline 789 & - & 35 & 204 & 54 & $4 \frac{1}{2}$ & 0 \\
\hline 790 & - & 35 & 185 & 57 & 1 & 0 \\
\hline 791 & - & 27 & 150 & 49 & 5 & 0 \\
\hline & & 92 & 05 & 53 & 1 & + \\
\hline
\end{tabular}

Group 104. Diet 60 without any addition:

$\begin{array}{lllrrrrr}711 & - & 25 & 70 & <10 & 60 & + & + \\ 712 & 18 & - & 54 & - & - & + & 0 \\ 713 & 52 & - & 290 & 34 & - & + & ++ \\ 714 & - & 41 & 99 & - & - & + & ++ \\ 715 & - & 46 & 172 & 10 & >12 & + & ++ \\ 716 & - & 33 & 88 & 15 & 10 & + & + \\ 717 & 46 & - & 158 & - & - & + & + \\ 718 & - & 19 & 48 & <10 & >30 & + & 0 \\ 719 & - & 27 & 46 & <8 & >500 & + & 0\end{array}$

Group 156. Diet $111+$ fresh tomato ad lib.:

$\begin{array}{llrrrcrl}978 & - & 34 & 190 & 50 & 1 & 0 & 0 \\ 979 & - & 32 & 225 & 50 & 1 \frac{1}{2} & 0 & 0 \\ 980 & - & 31 & 133 & 62 & 1 & ? & 0 \\ 981 & - & 3 & 35 & \overline{52} & 6 \frac{1}{2} & 0 & 0 \\ 982 & - & 34 & 125 & 52 & 15-3 & + & 0\end{array}$

Group 164. Diet $111+10 \%$ dried orange peel (outer layer):

$\begin{array}{lllllcll}1019 & - & 23 & 42 & - & 2 \frac{1}{2} & 0 & 0 \\ 1020 & - & 34 & 180 & 53 & 2 & 0 & ? \\ 1021 & 7 & - & 29 & - & -17 & 0 & 0 \\ 1022 & - & 32 & 182 & 39 & 17 & 0 & 0 \\ 1023 & - & 34 & 131 & 45 & 1 \frac{1}{2} & 0 & + \\ 1049 & - & 25 & 120 & 50 & 3 & 0 & 0 \\ 1050 & - & 25 & 111 & 50 & 10-15 & 0 & 0\end{array}$

Group 184. Diet 111 + fresh carrot ad lib.:

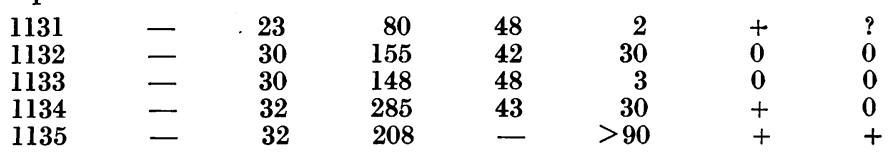


Table I (cont.).

Haemorrhages

\begin{tabular}{|c|c|c|c|c|c|}
\hline 110 & $\begin{array}{c}\text { Age in days } \\
\text { when }\end{array}$ & Weight & $\begin{array}{l}\text { Haemo- } \\
\text { globin }\end{array}$ & $\begin{array}{l}\text { Time of } \\
\text { clotting }\end{array}$ & $\begin{array}{l}\text { In the } \\
\text { lining } \\
\text { of the }\end{array}$ \\
\hline No. & Dead Killed & g. & (Sahli) & mins. & gizzard \\
\hline
\end{tabular}

Group 137. $20 \%$ dried calf-brain:

$\begin{array}{llllllll}887 & - & 23 & 92 & 48 & >30 & + & + \\ 888 & - & 30 & 81 & 30 & >30 & + & 0 \\ 889 & - & 28 & 67 & 40 & >60 & + & + \\ 890 & - & 19 & 66 & 10 & >60 & + & 0 \\ 891 & - & 31 & 136 & 52 & >60 & 0 & 0\end{array}$

Group 157. $20 \%$ dried ox-muscle:

\begin{tabular}{|c|c|c|c|c|c|c|}
\hline 984 & - & 35 & 130 & 45 & $>\mathbf{3 0}$ & 0 \\
\hline 985 & - & 23 & 175 & 40 & 6 & 0 \\
\hline 986 & - & 34 & 210 & 36 & $>300$ & $?$ \\
\hline 987 & - & 24 & 116 & 46 & 12 & + \\
\hline 988 & & 35 & 220 & 40 & 10 & 0 \\
\hline
\end{tabular}

Group 158. $20 \%$ dried adrenals (ox):

$\begin{array}{lrrrrrrl}989 & - & 33 & 112 & 55 & 3 \frac{1}{2} & 0 & 0 \\ 990 & - & 33 & 112 & 52 & 12 & 0 & 0 \\ 991 & 6 & - & 22 & - & >10 & ? & 0 \\ 992 & 5 & - & 28 & - & - & 0 & 0 \\ 993 & - & 9 & 41 & 50 & 15 & 0 & 0\end{array}$

Group 159. $20 \%$ dried ox-kidney :

$\begin{array}{rrrrrrrr}994 & - & 39 & 214 & 48 & 35 & 0 & 0 \\ 995 & - & 39 & 252 & 35 & 4 & 0 & 0 \\ 996 & 5 & - & 38 & - & - & 0 & 0 \\ 997 & - & 37 & 330 & 48 & 9 \frac{1}{2} & 0 & 0 \\ 998 & 6 & - & 40 & - & - & 0 & 0 \\ 1053 & - & 39 & 178 & 52 & 12 & 0 & 0 \\ 1054 & - & 32 & 187 & 44 & 23 & 0 & 0\end{array}$

Group 160. $20 \%$ dried ox-lung:

$\begin{array}{rrrrrrrr}999 & - & 41 & 172 & 30 & >200 & ? & + \\ 1000 & - & 41 & 150 & 30 & 28 & + & + \\ 1001 & - & 29 & 180 & 25 & >60 & 0 & ++ \\ 1002 & 40 & - & 170 & - & - & + & + \\ 1003 & - & 23 & 164 & 42 & 18 & 0 & ++\end{array}$

Group 161. $20 \%$ dried calf-thymus:

$\begin{array}{lrrrrrrr}1004 & - & 35 & 132 & 40 & >120 & 0 & + \\ 1005 & - & 38 & 191 & 47 & 60 & 0 & 0 \\ 1006 & - & 38 & 150 & 42 & 30 & 0 & 0 \\ 1007 & - & 38 & 170 & 51 & 16 & + & + \\ 1008 & - & 35 & 68 & 30 & 4 \frac{1}{2} & + & 0\end{array}$

Group 105. $20 \%$ dried hog-liver:

$\begin{array}{cccccccc}721 & - & 29 & 133 & 60 & 1 & 0 & 0 \\ 722 & 22 & - & 147 & - & - & 0 & 0 \\ 723 & 7 & - & 39 & - & - & 0 & 0 \\ 724 & - & 28 & 177 & 55 & 1 & 0 & 0 \\ 725 & - & 42 & 290 & 43 & 1 & 0 & 0 \\ 1164 & - & 26 & 140 & 55 & 1 & 0 & 0 \\ 1164 a & - & 31 & 251 & 53 & 16 & 0 & 0 \\ 1165 & - & 29 & 134 & 48 & 2 & 0 & 0 \\ 1166 & - & 31 & 154 & 50 & 5 & 0 & 0 \\ 1167 & - & 31 & 167 & 54 & 4 \frac{1}{2} & 0 & 0 \\ 1168 & - & 28 & 165 & 50 & 1-3 & 0 & ?\end{array}$

Fractions of HOG-LIVER.

Group 124. $20 \%$ dried hog-liver extracted with ether:

$\begin{array}{rrrrrrrr}821 & - & 24 & 150 & 46 & 20 & + & + \\ 822 & - & 26 & 153 & 50 & 10 & + & 0 \\ 823 & - & 18 & 93 & 47 & >1000 & + & + \\ .824 & - & 27 & 178 & 50 & >30 & 0 & ? \\ 825 & 24 & - & 138 & 52 & >60 & 0 & +\end{array}$


Table I (cont.).

\begin{tabular}{|c|c|c|c|c|c|c|c|}
\hline \multirow[b]{2}{*}{$\begin{array}{l}\text { Chick } \\
\text { No. }\end{array}$} & \multirow{2}{*}{\multicolumn{2}{|c|}{$\begin{array}{c}\begin{array}{c}\text { Age in days } \\
\text { when }\end{array} \\
\text { (n) }\end{array}$}} & \multirow[b]{2}{*}{$\begin{array}{l}\text { Weight } \\
\text { g. }\end{array}$} & \multirow{2}{*}{$\begin{array}{l}\text { Haemo- } \\
\text { globin } \\
\text { (Sahli) }\end{array}$} & \multirow{2}{*}{$\begin{array}{l}\text { Time of } \\
\text { clotting } \\
\text { mins. }\end{array}$} & \multirow{2}{*}{$\begin{array}{l}\text { In the } \\
\text { lining } \\
\text { of the } \\
\text { gizzard }\end{array}$} & \\
\hline & & Killed & & & & & $\begin{array}{l}\text { Else } \\
\text { whe }\end{array}$ \\
\hline
\end{tabular}

Group 139. Diet $124+3 \%$ hog-liver fat:

\begin{tabular}{|c|c|c|c|c|c|c|}
\hline 897 & - & 48 & 270 & 48 & 2 & 0 \\
\hline 898 & - & 47 & 243 & 45 & $3 \frac{1}{2}$ & 0 \\
\hline 899 & - & 29 & 138 & 50 & $1 \frac{1}{2}$ & 0 \\
\hline 900 & - & 26 & 120 & 55 & $4^{2}$ & 0 \\
\hline 901 & & & 310 & & 0 & . \\
\hline
\end{tabular}

Group 125. Diet $124+3 \%$ fatty acids from hog-liver fat (corresponding to $4 \cdot 7 \%$ fat):

$\begin{array}{llllllll}826 & - & 22 & 132 & 52 & >30 & 0 & + \\ 827 & - & 21 & 134 & 42 & >30 & +? & + \\ 828 & - & 23 & 146 & 42 & 5 & 0 & + \\ 829 & - & 20 & 110 & 53 & >60 & 0 & + \\ 830 & - & 21 & 121 & 38 & >30 & 0 & +\end{array}$

Group 126. Diet $124+0 \cdot 37 \%$ non-saponifiable matter from hog-liver fat (corresponding to $4 \cdot 4 \%$ fat):

$\begin{array}{llllllll}831 & - & 29 & 200 & 56 & 14 & 0 & 0 \\ 832 & - & 25 & 152 & 52 & 12 & 0 & 0 \\ 833 & - & 29 & 157 & 53 & 13 & 0 & 0 \\ 834 & - & 29 & 204 & 57 & 2 \frac{1}{2} & 0 & 0 \\ 835 & - & 29 & 188 & 59 & 4 & 0 & 0\end{array}$

Group 150. Diet $124+0 \cdot 075 \%$ of a fraction of the non-saponifiable matter sparingly soluble in ether (corresponding to $6 \%$ fat):

$\begin{array}{rrrrrrrr}948 & 23 & - & 123 & - & - & + & ++ \\ 949 & 24 & - & 185 & - & - & + & ++ \\ 950 & - & 29 & 120 & 35 & >60 & + & + \\ 951 & 25 & - & 120 & - & - & + & + \\ 952 & - & 11 & 55 & 52 & 7 & + & +\end{array}$

Group 151. Diet $124+0.4 \%$ sterol from hog-liver fat (corresponding to $6 \%$ fat):

$\begin{array}{llllllll}953 & - & 28 & 165 & 22 & 60 & + & + \\ 954 & 22 & & -151 & - & - & 0 & + \\ 955 & - & 28 & 136 & 32 & 75 & 0 & ++ \\ 956 & - & 20 & 132 & 30 & 15 & 0 & ++ \\ 957 & - & 28 & 105 & 8 & >60 & + & ++\end{array}$

Group 152. Diet $124+$ non-saponifiable matter from hog-liver fat freed from sterol and the sparingly soluble fraction (corresponding to $6 \%$ fat):

$\begin{array}{llllllll}958 & - & 28 & 220 & 53 & 1 & 0 & 0 \\ 959 & - & 30 & 185 & 52 & 6 & 0 & 0 \\ 960 & - & 30 & 165 & 55 & 2 & 0 & 0 \\ 961 & - & 30 & 195 & 57 & 15-6 & 0 ? & 0 \\ 962 & - & 28 & 230 & 55 & 1 & 0 & 0\end{array}$

Group 171. Diet 124 + xanthophyll fraction of the non-saponifiable matter (corresponding to $6 \%$ fat in the first 8 days, thereafter to $12 \%$ ):

$\begin{array}{llllllll}1060 & 24 & - & 115 & - & >30 & + & + \\ 1061 & - & 24 & 130 & 45 & >45 & + & 0 \\ 1062 & 24 & - & 124 & - & >60 & + & + \\ 1063 & 16 & - & 78 & - & 93 & + & + \\ 1064 & - & 31 & 120 & 55 & 140 & + & 0\end{array}$

Group 172. Diet 124 + carotene fraction of the non-saponifiable matter (corresponding to $6 \%$ fat in the first 8 days, thereafter to $12 \%$ ):

$\begin{array}{llllllll}1065 & - & 30 & 170 & 55 & 1 \frac{1}{2} & 0 & 0 \\ 1066 & - & 29 & 185 & 50 & 4 & 0 & 0 \\ 1067 & 23 & - & 137 & - & - & 0 & 0 \\ 1068 & - & 31 & 118 & 55 & 4 & 0 & 0 \\ 1069 & - & 31 & 120 & 50 & 1 \frac{1}{2} & 0 & 0\end{array}$


Table I (cont.). Haemorrhages

\begin{tabular}{|c|c|c|c|c|c|c|c|}
\hline \multirow[b]{2}{*}{$\begin{array}{l}\text { Chick } \\
\text { No. }\end{array}$} & \multicolumn{2}{|c|}{$\begin{array}{c}\begin{array}{c}\text { Age in days } \\
\text { when }\end{array} \\
\end{array}$} & \multirow{2}{*}{$\begin{array}{l}\text { Weight } \\
\text { g. }\end{array}$} & \multirow{2}{*}{$\begin{array}{l}\text { Haemo- } \\
\text { globin } \\
\text { (Sahli) }\end{array}$} & \multirow{2}{*}{$\begin{array}{l}\text { Time of } \\
\text { clotting } \\
\text { mins. }\end{array}$} & \multirow{2}{*}{$\begin{array}{l}\text { In the } \\
\text { lining } \\
\text { of the } \\
\text { gizzard }\end{array}$} & \multirow{2}{*}{$\begin{array}{l}\text { Else- } \\
\text { where }\end{array}$} \\
\hline & Dead & Killed & & & & & \\
\hline
\end{tabular}

Group 185. Diet $124+4 \%$ hog-liver fat which had been heated on a boiling water-bath for 12 hours in contact with air (as a 1-2 mm. deep layer):

$\begin{array}{llllllll}1136 & - & 30 & 220 & 58 & 2 & + & 0 \\ 1137 & - & 30 & 165 & 52 & 5 \frac{1}{2} & 0 & 0 \\ 1138 & - & 30 & 200 & 50 & 6 & ? & 0 \\ 1139 & - & 30 & 220 & 55 & 1 \frac{1}{2} & 0 & 0 \\ 1140 & - & 30 & 155 & 58 & 2 & 0 & 0\end{array}$

Additions of vitamins A, D and E.

Group 154. Diet $124+$ (extra) $4 \%$ cod-liver oil:

$\begin{array}{llllllll}968 & - & 27 & 140 & 29 & >60 & + & ++ \\ 969 & - & 27 & 112 & 32 & >60 & 0 & + \\ 970 & - & 13 & 84 & 52 & >120 & 0 & ++ \\ 971 & - & 24 & 125 & 50 & >60 & + & ++ \\ 972 & - & 24 & 105 & 40 & >60 & + & ++\end{array}$

Group 182. Diet 124 with halibut-liver oil instead of cod-liver oil (4\%) (1 g. of this oil contains 42,000 I.U. A and 2800 I.U. D):
$\begin{array}{lll}1121 & - & 27 \\ 1122 & 13 & - \\ 1123 & 30 & -30 \\ 1125 & - & 27\end{array}$
135
78
175
108
171
$\begin{array}{lr}\frac{35}{-} & -205 \\ \overline{38} & 230 \\ 38 & >230\end{array}$
$\begin{array}{cc}+ & 0 \\ + & ++ \\ + & ++ \\ 0 & 0 \\ + & 0\end{array}$

Group 175. Diet $124+$ daily doses of "vogan" (1 ml. vogan contains 120,000 I.U. A):

$\begin{array}{rrrrrr}1084 & - & 33 & 164 & 42 & 18 \\ 1085 & - & 33 & 188 & 43 & >240 \\ 1086 & - & 33 & 212 & 41 & >300 \\ 1087 & 15 & - & 50 & - & - \\ 1088 & - & 33 & 211 & 40 & >270\end{array}$

Group 178. Diet 124 + daily doses of carotene (Hoffmann La Roche):

$\begin{array}{lccc}+ & 0 & 4 \cdot 25 & 510,000 \\ 0 & 0 & \mathbf{4} \cdot 25 & 510,000 \\ + & + & \mathbf{4} \cdot 25 & 510,000 \\ + & ++ & 1 \cdot 1 & 132,000 \\ + & 0 & \mathbf{4} \cdot 25 & 510,000\end{array}$

Vogan

Total ml. I.U.

arotene

$\begin{array}{lllllclcl}1099 & - & 31 & 232 & 42 & 7 \frac{1}{2} & + & 0 & 9 \cdot 6 \\ 1100 & - & 28 & 124 & 38 & 18 & + & ++ & 8 \cdot 6 \\ 1101 & - & 31 & 212 & 48 & 12 & + & + & 9 \cdot 6 \\ 1102 & 23 & - & 175 & - & - & + & + & 3 \cdot 05 \\ 1103 & - & 28 & 121 & 38 & >120 & 0 & ++ & 8 \cdot 6\end{array}$

Group 163. Diet 124 + daily doses of "vigantol" (1 ml. vigantol contains 15,000 I.U. D):

$\begin{array}{llllllllll}1014 & - & 34 & 175 & 48 & >120 & + & + & 0 \cdot 21 & 3150 \\ 1015 & - & 28 & 152 & 30 & >60 & + & + & 0 \cdot 15 & 2250 \\ 1016 & 33 & - & 190 & 40 & >60 & + & + & 0 \cdot 20 & 3000 \\ 1017 & - & 34 & 200 & 52 & >120 & + & + & 0 \cdot 21 & 3150 \\ 1018 & - & 34 & 110 & 50 & 115 & + & + & 0 \cdot 21 & 3150\end{array}$

Group 155. Diet $124+4 \%$ wheat germ oil:

$\begin{array}{llllllll}973 & - & 34 & 218 & 50 & 60 & + & + \\ 974 & - & 6 & 20 & - & -1 & - & 0 \\ 975 & - & 42 & 375 & 50 & 60 & 0 & 0 \\ 976 & - & 31 & 261 & 50 & >60 & + & + \\ 977 & - & 32 & 233 & 45 & >60 & 0 & 0\end{array}$

Group 148. Diet $60+10 \%$ wheat germ oil:

\begin{tabular}{|c|c|c|c|c|c|c|c|}
\hline 938 & 37 & - & 110 & 58 & $>\mathbf{3 0}$ & + & + \\
\hline 939 & - & 19 & 39 & 10 & $>60$ & + & 0 \\
\hline 940 & - & 44 & 150 & 35 & 27 & + & + \\
\hline 941 & - & 44 & 120 & 35 & 35 & + & + \\
\hline & & 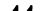 & 175 & 4 & 115 & + & $?$ \\
\hline
\end{tabular}


Table I (cont.).

Haemorrhages

\begin{tabular}{|c|c|c|c|c|c|c|}
\hline & $\begin{array}{l}\text { Age in days } \\
\text { when }\end{array}$ & & Haemo- & Time of & In the & \\
\hline $\begin{array}{l}\text { Chick } \\
\text { No. }\end{array}$ & Killed & $\begin{array}{l}\text { Weight } \\
\text { g. }\end{array}$ & $\begin{array}{l}\text { (Sabin } \\
\text { (Sahli) }\end{array}$ & $\begin{array}{l}\text { clotting } \\
\text { mins. }\end{array}$ & $\begin{array}{l}\text { of the } \\
\text { gizzard }\end{array}$ & $\begin{array}{l}\text { Else- } \\
\text { where }\end{array}$ \\
\hline
\end{tabular}

Group 186. Diet $111+24 \%$ wheat germ oil:

$\begin{array}{llllllll}1141 & - & 30 & 143 & 58 & 18 & + & 0 \\ 1142 & 15 & - & 50 & 5 & 1 & 0 & 0 \\ 1143 & - & 30 & 170 & 51 & 1 \frac{1}{2} & + & 0 \\ 1144 & - & 30 & 200 & 52 & 12 & + & 0 \\ 1145 & - & 30 & 160 & 54 & 2 & + & 0\end{array}$

Different fats and oIls.

Group 110. Diet $60+10 \%$ butter fat:

$\begin{array}{lllrlrrr}\mathbf{7 4 6} & - & 31 & 80 & <8 & 90 & + & 0 \\ \mathbf{7 4 7} & - & 38 & 138 & 45 & 7 & 0 & 0 \\ \mathbf{7 4 8} & - & 38 & 144 & 31 & 11 & + & + \\ \mathbf{7 4 9} & \mathbf{2 1} & - & 64 & - & - & + & + \\ \mathbf{7 5 0} & - & 21 & \mathbf{7 2} & \mathbf{5 0} & >\mathbf{6 0} & ? & 0\end{array}$

Group 128. Diet $60+10 \%$ cod-liver oil:

$\begin{array}{llllllll}842 & - & 20 & 47 & 20 & >120 & + & + \\ 843 & 17 & - & 38 & - & - & + & + \\ 844 & - & 20 & 51 & 26 & >60 & + & + \\ 845 & - & 18 & 70 & 31 & >30 & + & + \\ 846 & - & 13 & 38 & 10 & >10 & + & 0\end{array}$

Group 130. Diet $60+10 \%$ soya bean oil:

$\begin{array}{llllllll}852 & - & 27 & 112 & 50 & 5 & + & + \\ 853 & - & 23 & 113 & 58 & 7 & ? & + \\ 854 & - & 42 & 168 & 53 & 5 & + & 0 \\ 855 & 23 & - & 73 & - & - & 0 & ? \\ 856 & - & 42 & 191 & 55 & 10 & 0 & 0\end{array}$

Group 131. Diet $60+10 \%$ copra oil:

$\begin{array}{llllrrrr}857 & - & 13 & 41 & <10 & >30 & + & 0 \\ 858 & - & 23 & 74 & 44 & 120 & ? & 0 \\ 859 & 12 & - & 37 & - & - & + & 0 \\ 860 & - & 23 & 97 & 50 & 27 & 0 & 0 \\ 861 & - & 23 & 82 & 50 & 5 & ? & 0\end{array}$

Group 140. Diet $60+10 \%$ hog-liver fat:

$\begin{array}{llllllll}902 & - & 28 & 45 & 30 & \cdot 2 \frac{1}{2} & 0 & 0 \\ 903 & - & 28 & 67 & 56 & 1 & 0 & 0 \\ 904 & - & 24 & 39 & 50 & 2 \frac{1}{2} & ? & 0 \\ 905 & - & 28 & 89 & 54 & 2 & 0 & 0 \\ 906 & - & 28 & 68 & 52 & 4 & 0 & 0\end{array}$

Group 14l. Diet $60+10 \%$ rape oil:

$\begin{array}{lllrlclc}907 & - & 37 & 178 & 50 & 28 & 0 & 0 \\ 908 & - & 37 & 140 & 53 & 2 \frac{1}{2} & 0 & 0 \\ 909 & 29 & - & 50 & - & - & ? & + \\ 910 & - & 36 & 130 & 38 & >14 & ? & ++ \\ 911 & - & 37 & 154 & 55 & 95 & 0 & 0\end{array}$

Group 142. Diet $60+10 \%$ linseed oil:

$\begin{array}{lrlrrrrc}912 & - & 28 & 55 & 18 & 20 & + & 0 \\ 913 & - & 28 & 83 & 25 & 30 & + & 0 \\ 914 & - & 24 & 49 & <10 & >60 & + & ++ \\ 915 & - & 28 & 89 & 47 & - & + & + \\ 916 & - & 26 & 58 & <5 & >70 & + & 0\end{array}$

Group 191. Diet $124+10 \%$ lard:

$\begin{array}{lrlrrrrr}1169 & - & 30 & 199 & 55 & 24 & + & 0 \\ 1170 & - & 30 & 153 & 47 & >110 & + & 0 \\ 1171 & - & 30 & 149 & 48 & 22 & ? & 0 \\ 1172 & 9 & - & 64 & - & - & + & ++ \\ 1173 & - & 30 & 149 & 42 & >150 & + & 0\end{array}$


Table I (cont.).

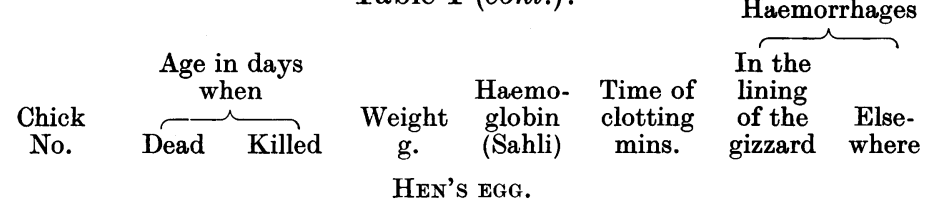

Group 54. Diet 49* $+10 \%$ fresh egg-yolk:

$\begin{array}{rrrrrrrr}431 & - & 49 & 241 & - & - & + & ++ \\ 432 & - & 52 & 252 & - & - & 0 & ++ \\ 433 & - & 20 & 64 & - & - & + & + \\ \mathbf{4 3 4} & - & 55 & \mathbf{4 5 2} & \mathbf{6 4} & - & ? & 0 \\ \mathbf{4 3 5} & - & 55 & 352 & \mathbf{4 7} & - & 0 & ++\end{array}$

Group 55. Diet $49+10 \%$ fresh egg-white:

$\begin{array}{llllllll}436 & - & 18 & 43 & - & - & + & + \\ 437 & - & 18 & 42 & - & - & + & 0 \\ 438 & - & 18 & 54 & - & - & + & ++ \\ 439 & - & 18 & 53 & - & - & + & ++ \\ 440 & 18 & - & 57 & - & - & ? & +\end{array}$

Group 56. Diet 49 in which $20 \%$ caseinogen was replaced by $20 \%$ commerical desiccated egg-white:

$\begin{array}{llllllll}441 & - & 39 & 86 & - & - & + & 0 \\ 442 & - & 27 & 66 & - & - & + & 0 \\ 443 & - & 39 & 73 & - & - & + & 0 \\ 444 & - & 24 & 53 & - & - & + & 0 \\ 445 & - & 31 & 45 & - & - & + & ?\end{array}$

Group 167. Diet 60 in which $25 \%$ sucrose was replaced by $25 \%$ commerical desiccated egg-yolk:

$\begin{array}{lllllcll}1034 & - & 38 & 270 & 52 & \frac{1}{2}-4 \frac{1}{2} & 0 & 0 \\ 1035 & - & 38 & 220 & 50 & 8 \frac{1}{2}-28 & 0 & 0 \\ 1036 & - & 39 & 295 & 45 & 4 & ? & 0 \\ 1037 & - & 38 & 300 & 50 & 8 \frac{1}{2}-28 & 0 & 0 \\ 1038 & - & 38 & 322 & 55 & 13 \frac{1}{2} & 0 & 0 \\ 1082 & - & 33 & 322 & 55 & 7 & 0 & 0\end{array}$

* Dam and Schönheyder [1934].

\section{RESULts AND DISCUSSION.}

Table I shows clearly that certain cereals and seeds will protect against the disease to a large extent whilst others are practically ineffective. Hemp seed appears to be particularly protective whilst unpolished rice, sunflower seed, yellow corn and rye are nearly valueless. The other cereals which have been tested appear to occupy a middle position.

Certain vegetables such as tomatoes, kale, orange peel etc. appear to have a fairly good action against the disease.

Among the organs so far tested, hog-liver is the most potent source of the active principle, while calf-brain, ox-lung and muscle are extremely poor. Oxkidney, adrenals and calf-thymus occupy a middle position.

The hen's egg does not appear to be a very rich source, but the yolk will no doubt afford protection when given in large quantities.

All symptoms-clotting time, haemorrhages in the gizzard and other organs and haemoglobin content-are taken into consideration in estimating the condition of the animals, since at the present state of the investigation, the possibility that certain factors in the food may render the gizzard more resistant or may counteract the tendency to haemorrhage which is in turn due to the retarded clotting, has not been excluded. It is also possible that other factors or properties of the food may reduce the resistance of the gizzard or favour the 
occurrence of spontaneous haemorrhages. ${ }^{1}$ Extremely retarded growth will undoubtedly counteract spontaneous haemorrhages.

It is not probable that the low haemoglobin values which are found in animals with large haemorrhages have anything to do with lack of copper, since similar results are obtained when the diet contains $10 \%$ marmite. 1 g. marmite has been found to contain about $0.06 \mathrm{mg}$. $\mathrm{Cu}$.

The results from groups 105, 124, 139 and 140 clearly demonstrate that the active principle in hog-liver fat can be extracted with ether. Corresponding experiments with hemp seed-groups 97, 120 and 132-lead to the conclusion that the ether extract of hemp seed is protective and that the action of the extracted seed is diminished but not entirely lost, thus suggesting that the active principle of hemp seed-like the carotene of green leaves-is not altogether in association with the fat. A series of other fats and oils was tested but none of them was found to be very potent.

McFarlane et al. [1931] have made the observation that chicks reared on an artificial diet with ether-extracted meat meal or ether-extracted white fish meal as the source of protein suffered from a tendency to haemorrhages whilst this was not the case when the meat meal or fish meal was not extracted with ether. This is in line with the results of the present paper. McFarlane et al. reported, however, that they could not observe haemorrhages when caseinogen, extracted or non-extracted, was used as protein. This latter statement is incompatible with the findings of Dam [1929; 1930] and Dam and Schönheyder [1934] as well as with the present experiments in which the diets contain caseinogen.

Fractionation of hog-liver fat into fatty acids-group 125-and non-saponifiable matter-group 126-revealed the fact that the antihaemorrhagic factor is to be found in the non-saponifiable fraction, and by further fractionationgroups 150, 151 and 152-the factor was found in the easily soluble non-sterol fraction, the sterols and a fraction sparingly soluble in ether being inactive. From a solution in light petroleum the factor could not be removed by repeated shaking with $90 \%$ methyl alcohol-groups 171 and 172 . In this respect the antihaemorrhagic factor behaves like vitamin E [Olcott and Mattill, 1931] and differs from vitamin A [Wolff et al., 1930]. (The paper of the last-mentioned authors deals with the partition of vitamin A between light petroleum and ethyl alcohol, but, according to a personal communication from Prof. Wolff, it has been established that methyl alcohol behaves in the same manner.)

Addition of very large amounts of vitamins $A$ and $D$ to the diet does not prevent the disease-group 154, extra $4 \%$ cod-liver oil-group 182, replacement of the cod-liver oil of the basal diet by halibut-liver oil containing 42,000 I.U. of $A$ and 2800 I.U. of D per g. whereby the amounts of vitamins A and D are raised materially - group 175, addition of the concentrated vitamin A preparation "vogan"-group 163, addition of pure vitamin D in sesame oil "vigantol".

Commercial carotene (Hoffmann La Roche), which according to the statement of the firm contains $70-80 \% \beta$ - and 30-20\% $\alpha$-plus traces of $\gamma$-carotene, does not prevent the disease when given in quantities up to $9 \mathrm{mg}$. in 1 month.

1 Certain food components, such as desiccated hog-liver, hog-liver fat, large amounts of cod-liver oil may produce symptoms resembling polyneuritis (3 animals in group 105, 3 in group 125, 2 in group 139). This polyneuritic condition is often accompanied by multiple minute haemorrhages in the cerebellum. Such haemorrhages, however, appear to be independent of the clotting time and general haemorrhagic condition since they may appear in groups where clotting time is normal and when no haemorrhages occur in other organs. The origin of these haemorrhages and of the polyneuritic symptoms is being studied further. Polyneuritic chicks are ligble to traumatic haemorrhages than others, owing to their convulsive movements 
According to Brockman and Völker [1934] hemp seed, which is very active, is practically devoid of carotene. The conclusion is therefore justified that the antihaemorrhagic vitamin cannot be identical with vitamins $\mathrm{A}$ or $\mathrm{D}$ or with $\alpha$ - or $\beta$-carotene. The antihaemorrhagic vitamin bears some resemblance to vitamin E. An experiment-group 185-in which the liver fat had been heated in a boiling water-bath for 12 hours in a 1-2 mm. thick film, showed that the factor is not destroyed to any great extent under these circumstances. According to Evans [1932] vitamin E will resist such treatment. On partition between light petroleum and $90 \%$ methyl alcohol the vitamin behaves, as mentioned, like vitamin $\mathrm{E}$. It appears that a loss occurs during the saponification.

Freshly prepared wheat germ oil, however, did not protect when given at a level of 4 or $10 \%$ in the diet, and $24 \%$ yielded an incomplete protectiongroups 155,148 and 186 . $62 \%$ wheat germ was rather inactive-group 86 . Owing to the fact that such large amounts of wheat germ oil are insufficient to prevent the disease while $3-4 \%$ of hog-liver fat is entirely protective, it must be considered very unlikely that the antihaemorrhagic vitamin is identical with vitamin $\mathbf{E}$.

It is therefore suggested that the term vitamin $K$ be used for the antihaemorrhagic factor until its chemical nature has been elucidated.

Comparison of the weights of the animals in groups 105, 124 and 172 appears to indicate that vitamin $\mathbf{K}$ does not promote growth.

The question whether the lack of this vitamin will produce the same symptoms in other animals than chicks is under investigation.

\section{SUMMARY.}

1. The nature and distribution of the antihaemorrhagic vitamin of the chick have been investigated.

2. The vitamin is fat-soluble and one of its richest sources is hog-liver fat, whilst cod-liver oil is practically devoid of it. The hen's egg contains the vitamin in the yolk but is apparently not an extremely rich source. In the vegetable kingdom hemp seed and certain vegetables are good sources, yellow corn, unpolished rice and sunflower seeds are very poor.

3. The vitamin occurs in the easily soluble non-sterol fraction of the unsaponifiable matter.

4. It cannot be identical with vitamin $A$ or $D$ because large amounts of these vitamins in the diet are completely ineffective in preventing the disease. It bears some resemblance to vitamin $\mathrm{E}$ with respect to solubility and resistance to heating in air, but is held to be different from $\mathrm{E}$ because large quantities of wheat germ and wheat germ oil do not afford complete protection against the disease.

5. It is suggested that the term vitamin $K$ be used for the antihaemorrhagic factor.

6. The further chemical nature and the possible importance of the vitamin for other animals than the chick are under investigation.

Thanks are due to Medicinalco, Mfg. Co., for kindly supplying dried organs, liver fat etc., and to Dr Sk. V. Gudjonsson, Statens Vitaminlaboratorium, for a standardised sample of halibut-liver oil. 


\section{REFERENCES.}

Brockmann and Völker (1934). Z. physiol. Chem. 224, 193.

Dam (1929). Biochem. Z. 215, 475.

- (1930). Biochem. Z. 220, 258. and Schönheyder (1934). Biochem. J. 28, 1355.

Evans (1932). J. Amer. Med. Assoc. 99, 469.

McFarlane, Graham and Richardson (1931). Biochem. J. 25, 358.

Olcott and Mattill (1931). J. Biol. Chem. 93, 61.

Schönheimer and Dam (1933). Z. physiol. Chem. 215, 59.

Schönheyder (1935). Nature, 135, 653.

Wolff, Overhoff and van Eckelen (1930). Deutsch. med. Woch. 56, 1428. 